\title{
Metastable CdTe@HgTe core@shell Nanostructures Obtained by Partial Cation Exchange Evolve into Sintered CdTe films Upon Annealing
}

Irene Rosina ${ }^{\mathrm{a}, \mathrm{b}}$, Beatriz Martin-Garciac, Davide Spirito ${ }^{\mathrm{d}}$, Zhiya Dang ${ }^{\mathrm{a}}$, Graziella Gariano ${ }^{\mathrm{a}}$, Sergio Marras ${ }^{\mathrm{e}}$, Mirko Prato ${ }^{\mathrm{e}}$, Roman Krahne ${ }^{\mathrm{d}}$, Luca De Trizio ${ }^{\mathrm{a}}$ and Liberato Manna ${ }^{\mathrm{a}}$

${ }^{a}$ Nanochemistry Department, ' Graphene Labs, dOptoelectronics, e Materials Characterization Facility, Istituto Italiano di Tecnologia (IIT), via Morego 30, Genova, Italy

bDipartimento di Chimica e Chimica Industriale, Università degli Studi di Genova, Via Dodecaneso 31, 16146 Genova, Italy

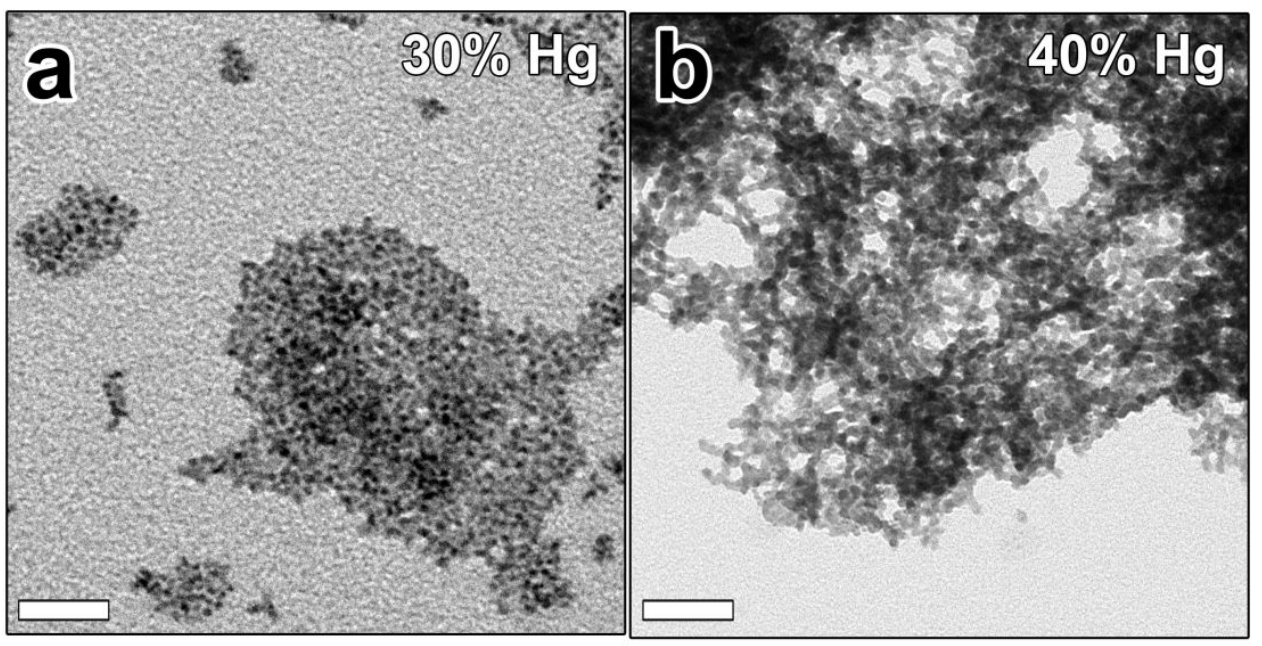

Figure S1. TEM images of CdTe-Hg NCs obtained by partial CE with $\mathrm{Hg}^{2+}$ cations employing $\mathrm{Hg} / \mathrm{Cd}$ feed ratios of (a) $30 \%$ and (b) $40 \%$. The scalebars are $50 \mathrm{~nm}$. 


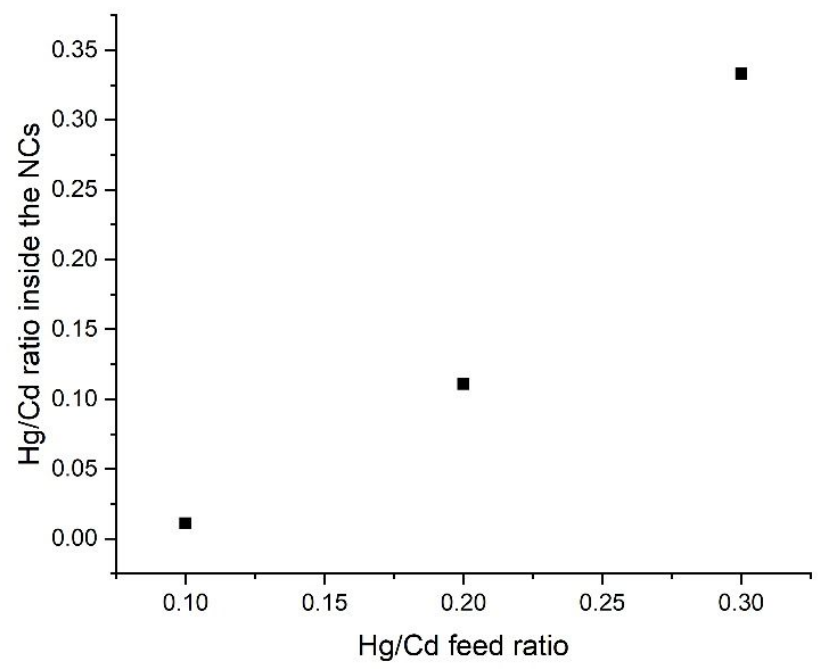

Figure S2. $\mathrm{Hg} / \mathrm{Cd}$ ratio inside the NCs (measured via ICP analysis) as a function of the $\mathrm{Hg} / \mathrm{Cd}$ feed ratio employed in the CE experiments. 

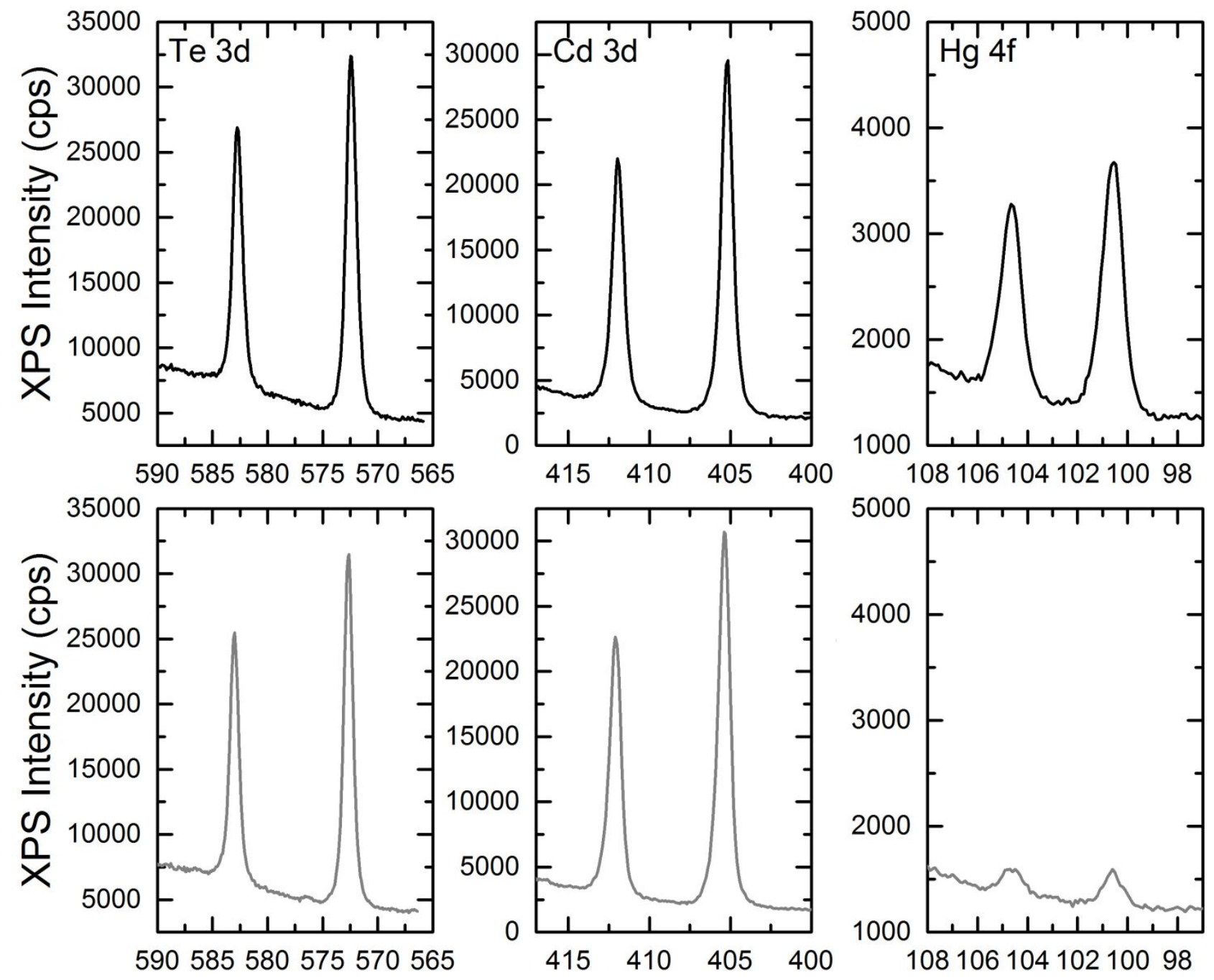

Bind. En. (eV)

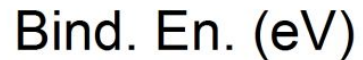

Bind. En. (eV)

Figure S3. XPS spectra of CdTe-Hg 10\% NCs before (upper panels) and after annealing (lower panels). Peak positions: Te $3 \mathrm{~d}_{5 / 2}$ at $(572.5 \pm 0.2) \mathrm{eV}, \mathrm{Cd} 3 \mathrm{~d}_{5 / 2}$ at $(405.3 \pm 0.2) \mathrm{eV}$ and $\mathrm{Hg} 4 \mathrm{f}_{7 / 2}$ at $(100.6 \pm 0.2) \mathrm{eV}$, indicating the presence of $\mathrm{Te}^{2-}, \mathrm{Cd}^{2+}$ and $\mathrm{Hg}^{2+}$ in the sample [NIST X-ray Photoelectron Spectroscopy Database, Version 4.1 (National Institute of Standards and Technology, Gaithersburg, 2012); http://srdata.nist.gov/xps/]. Relative atomic ratios were determined using the integrated area under $\mathrm{Cd}_{3} \mathrm{~d}$, Te $3 \mathrm{~d}$ and $\mathrm{Hg} 4 \mathrm{f}$ principal peaks. 


\section{Computational model:}

A zincblende $\mathrm{Cd}_{1028} \mathrm{Te}_{919}$ nanocrystal of $\sim 4.2 \mathrm{~nm}$ in diameter has been constructed. This model features

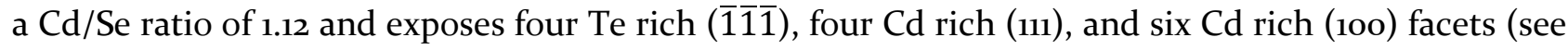
Figure SXX). By replacing all the superficial $\mathrm{Cd}$ atoms by $\mathrm{Hg}$ ones, the stoichiometry becomes $\mathrm{Cd}_{724} \mathrm{Hg}_{304} \mathrm{Te}_{919}$. . Our model indicates that a HgTe monolayer can be achieved by $\mathrm{CE}$ only when substituting almost $40 \%$ of $\mathrm{Cd}^{2+}$ cations that is, when achieving $\mathrm{Hg} / \mathrm{Cd}$ ratio of 0.42 .

\section{$\mathrm{CdTe}$}

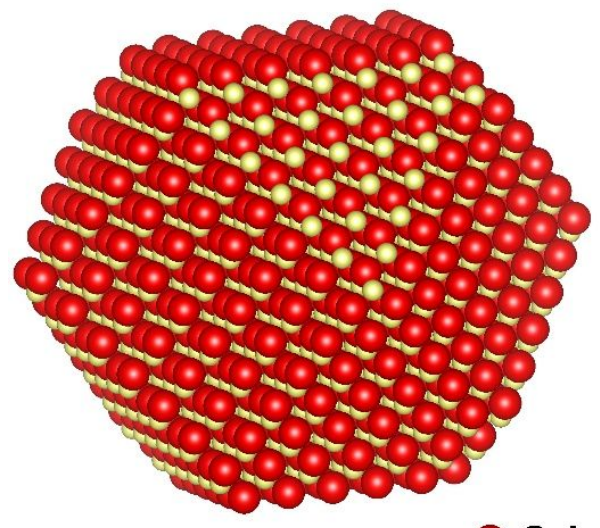

CdTe@HgTe (1ML)

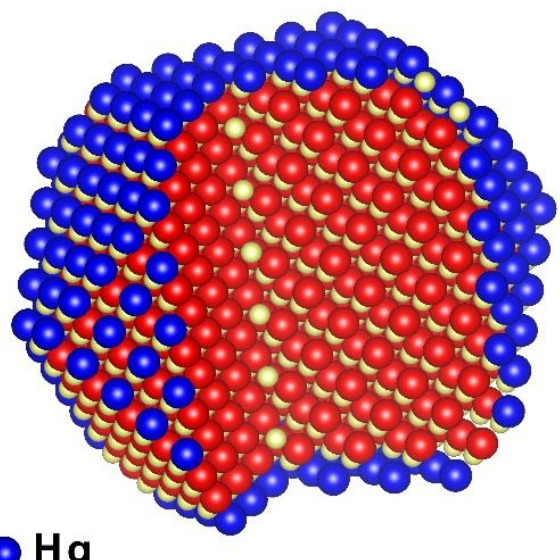

$\mathrm{Cd} \odot \mathrm{Te} \odot \mathrm{Hg}$

Figure $\mathbf{S}_{4}$. Models of zincblende $4.2 \mathrm{~nm} \mathrm{CdTe}$ and CdTe@HgTe nanocrystals, the latter being characterized by one HgTe monolayer thick shell.

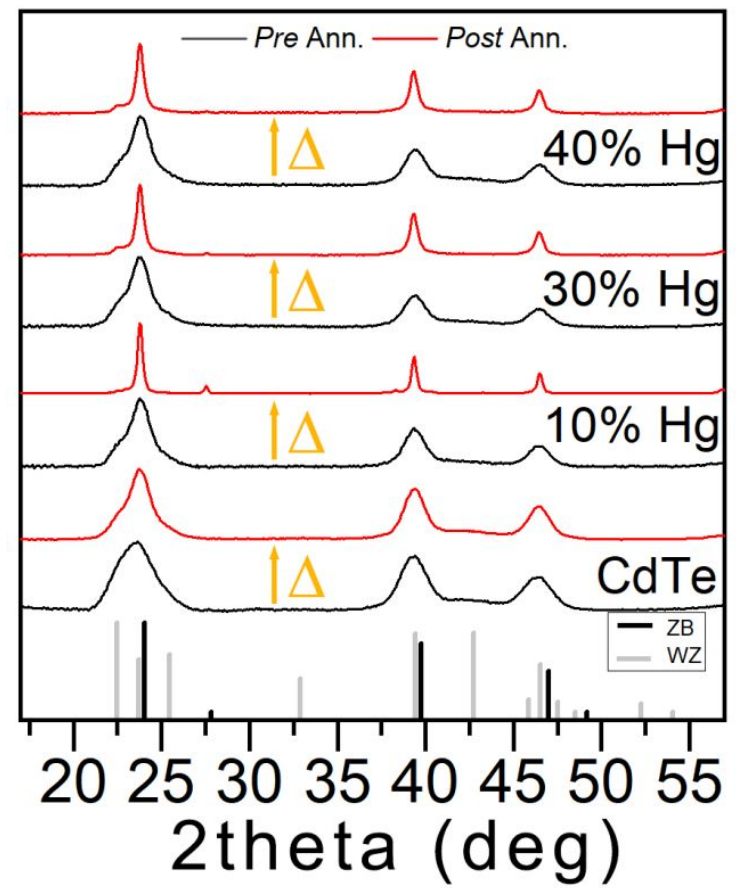

Figure $\mathrm{S}_{5}$. XRD patterns of CdTe and CdTe-Hg NC samples subjected to a thermal treatment under $\mathrm{N}_{2}$ from RT (black curves) to $200^{\circ} \mathrm{C}$ (red curves). The bulk reflections of WZ and ZB CdTe structures are reported by means of gray and black bars, respectively. 

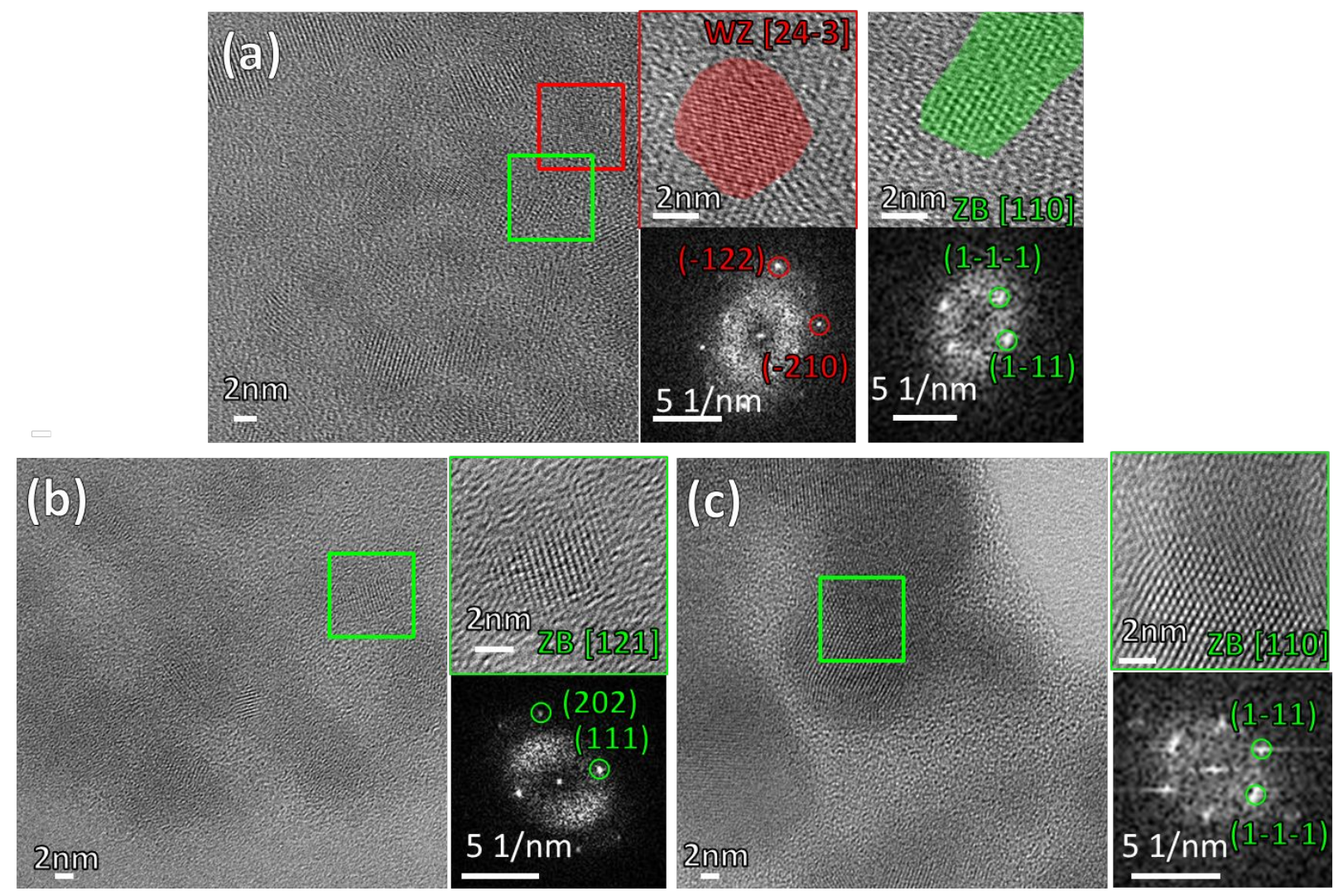

Figure S6. HRTEM images of CdTe-Hg $10 \%$ NCs before (a) and after annealing at $200^{\circ} \mathrm{C}(\mathrm{b}, \mathrm{c})$ with the corresponding FFT.

Wide view HRTEM before annealing

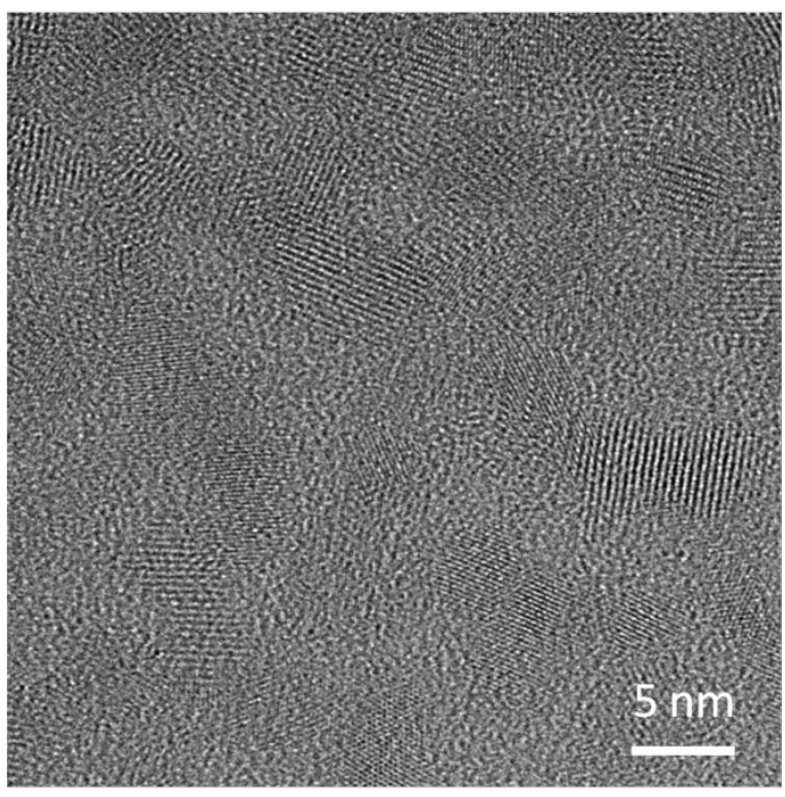

Wide view HRTEM after annealing

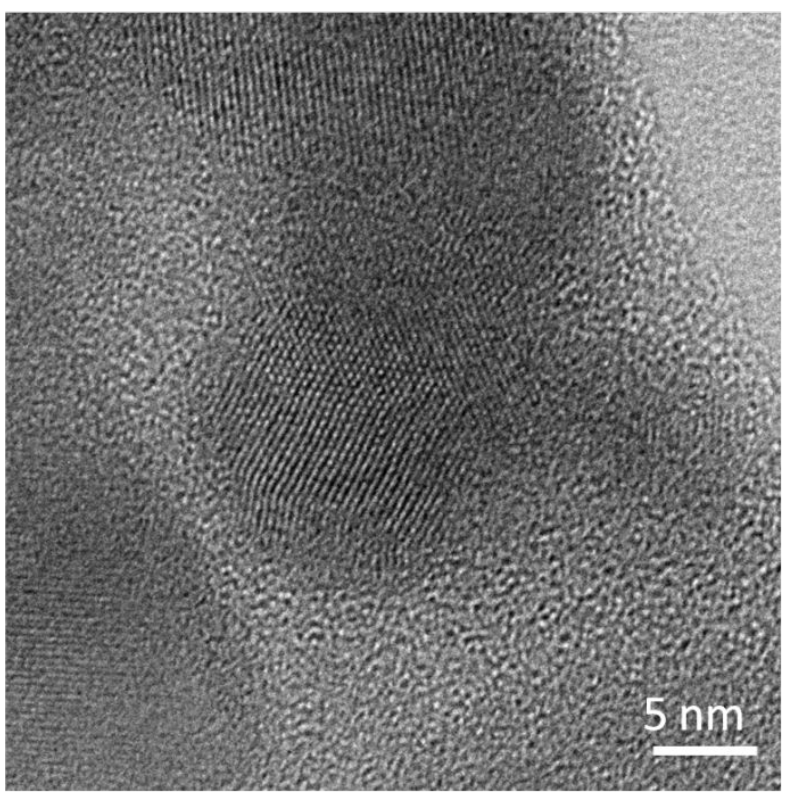

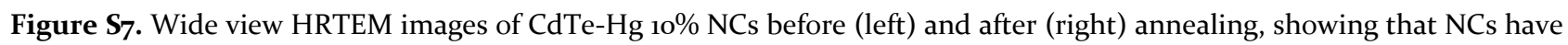
merged into larger crystallites. 

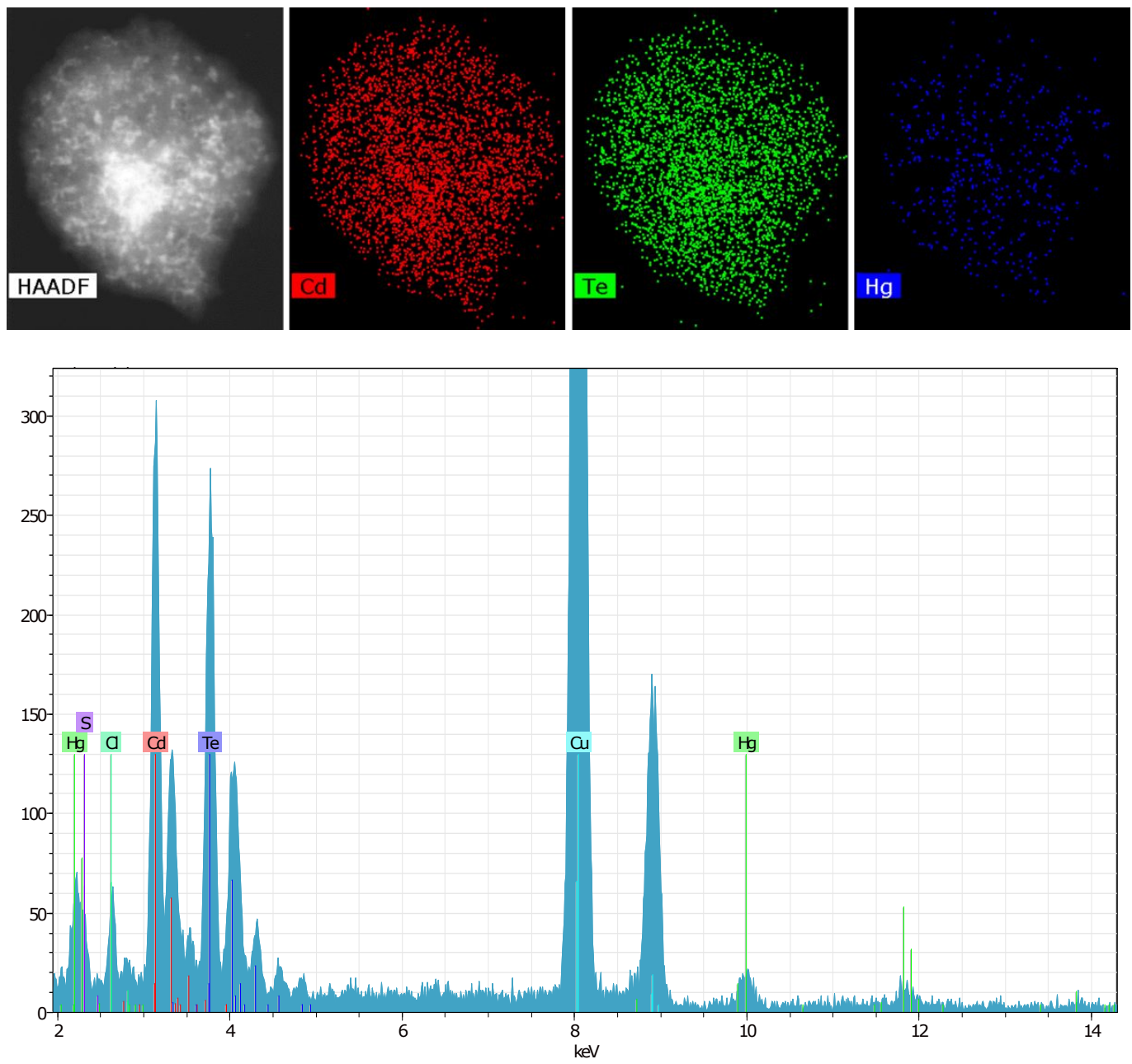

Figure S8. STEM-EDX mapping of CdTe@HgTe 10\% NCs (top panels) with the corresponding measured spectrum (bottom panel). The composition of the NC ensemble was measured to be $\mathrm{CdHg}_{0.1} \mathrm{Te}$. 

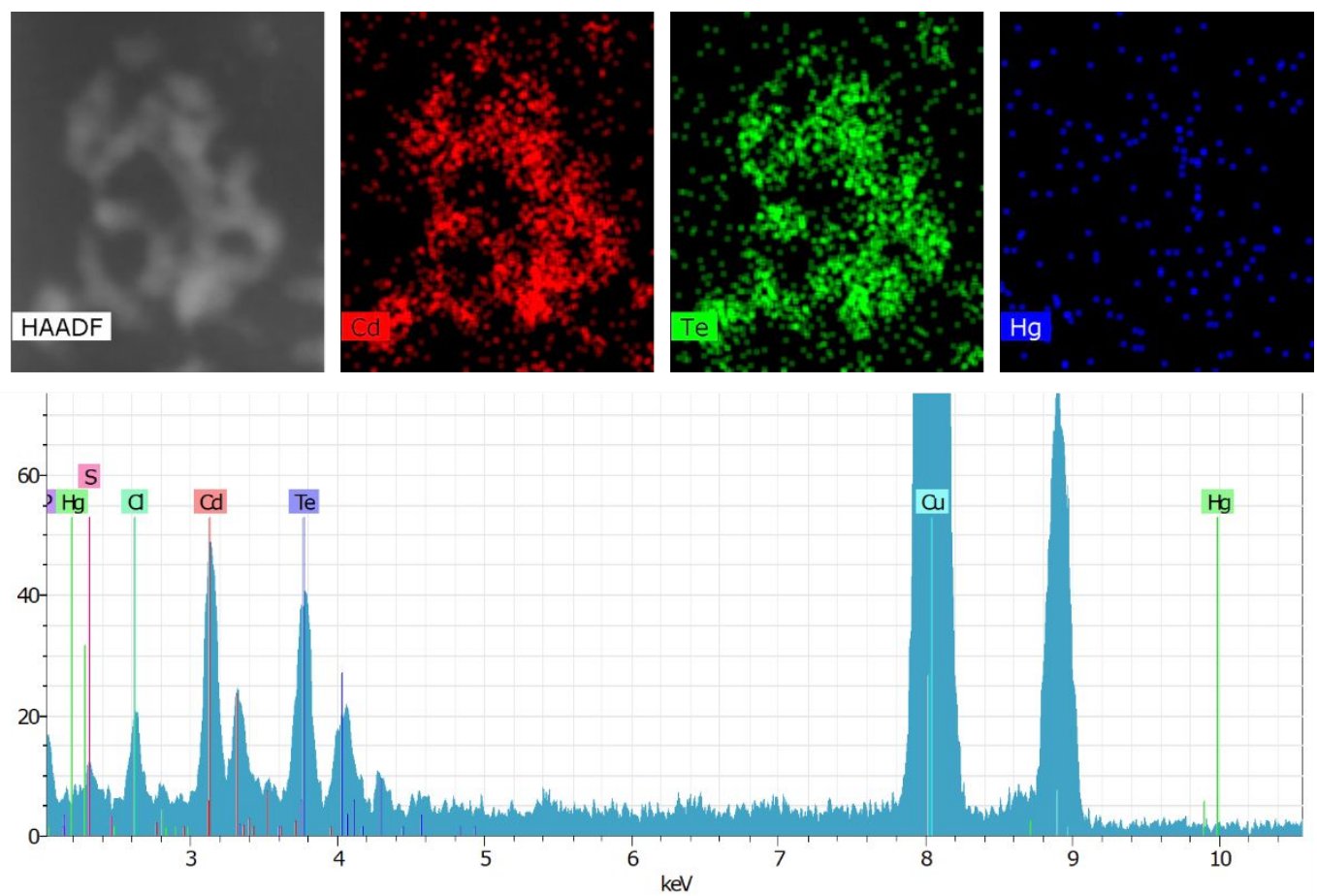

Figure S9. STEM-EDX mapping of CdTe-Hg $10 \% \mathrm{NCs}$ after annealing at $200^{\circ} \mathrm{C}$ (top panels) with the corresponding spectrum (bottom panel). The composition of the NC ensemble was measured to be $\mathrm{Cd}_{1.03} \mathrm{Te}$. 

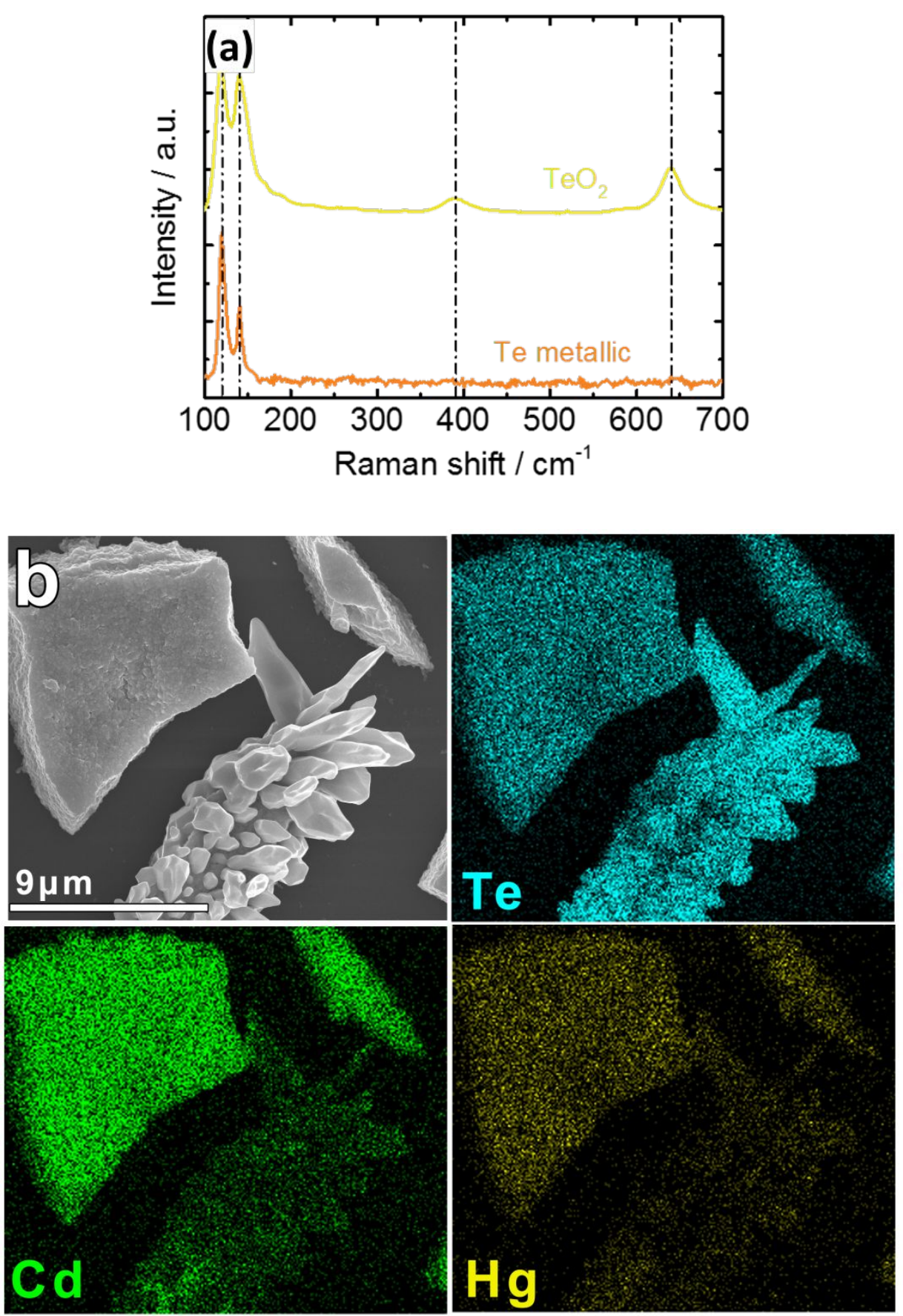

Figure S1o. (a) Raman spectra of metallic Te characterized by two peaks at 120 and $140 \mathrm{~cm}^{-1}$ and $\mathrm{TeO}_{2}$ with additional peaks at 390 and $640 \mathrm{~cm}^{-1}$. (b) SEM image of a section of a film of CdTe-Hg $10 \% \mathrm{NCs}$ after annealing at $200^{\circ} \mathrm{C}$. The rod like structure present in the SEM image is composed of Te only, as emerged from the corresponding SEM-EDX measurements. 


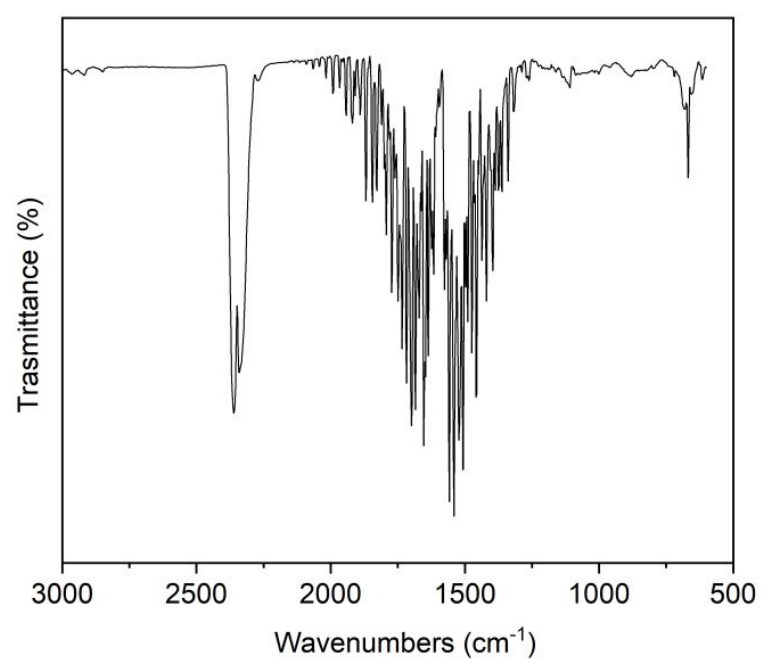

Figure S11. FTIR spectrum of a "blank" KBr pellet employed in the FTIR analyses of CdTe and CdTe-Hg NC samples.
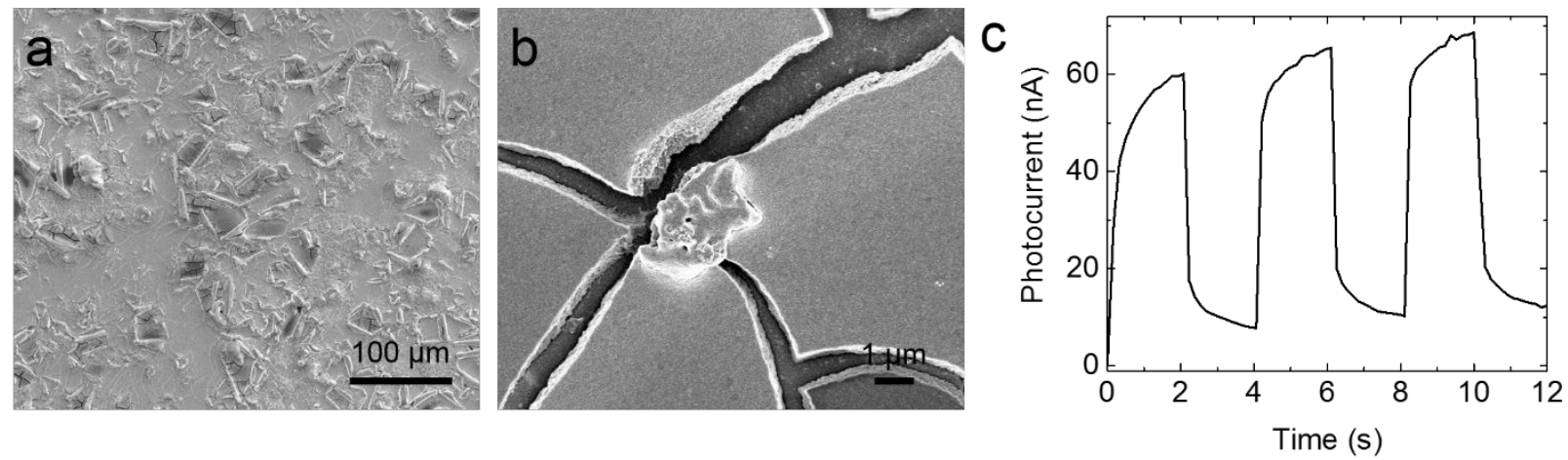

Figure S12. (a, b) Representative SEM images of a CdTe film obtained by spin coating CdTe-Hg $10 \%$ NCs and by annealing at $200^{\circ} \mathrm{C}$. (c) Time trace of the photocurrent while the light was switched on and off, at room temperature in air under white light (LED ILH-ONo9-VA, $90 \mathrm{~mW} / \mathrm{cm}^{2}$ ). 


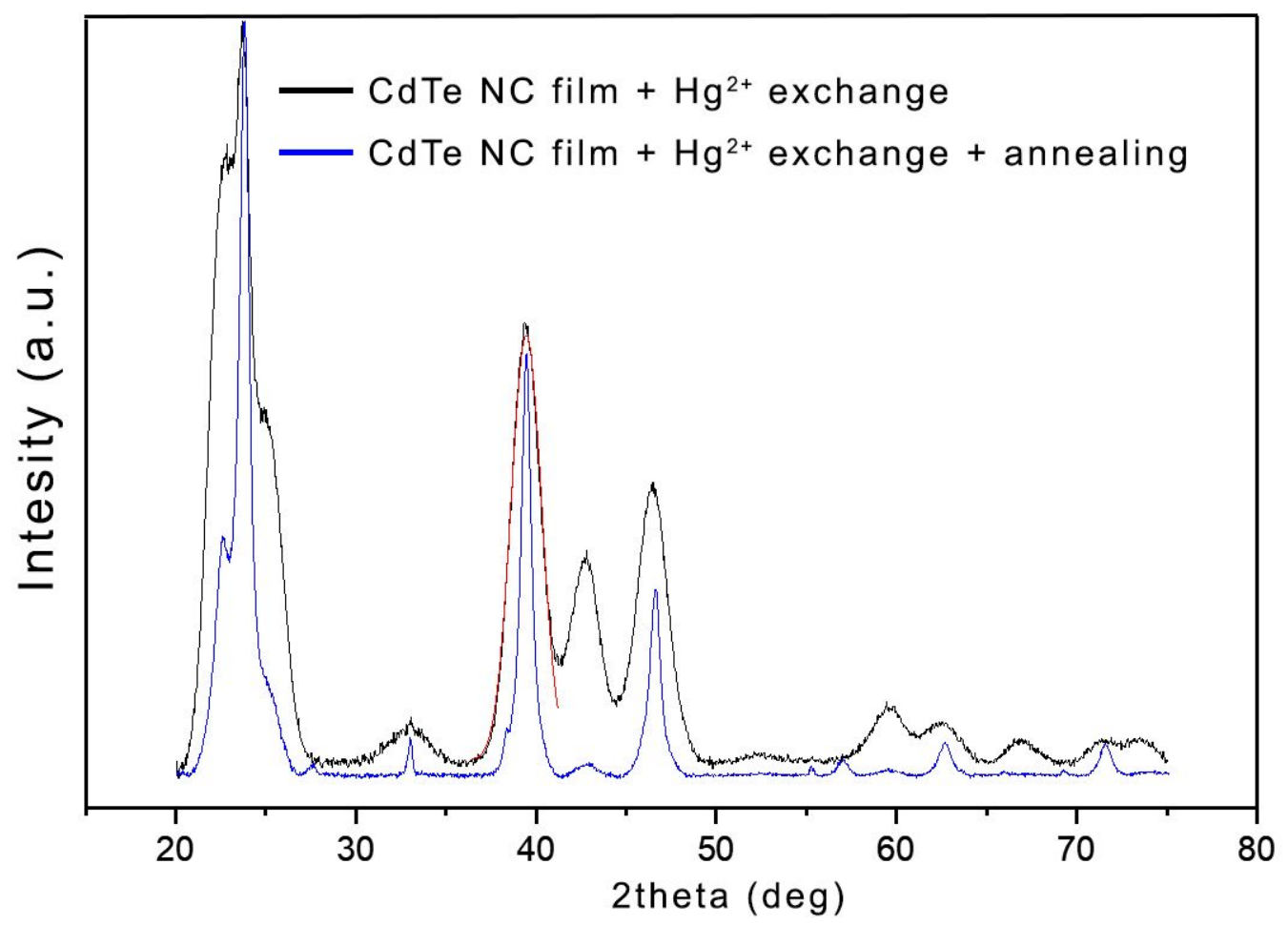

Figure S13. (a) XRD pattern of a film obtained by first depositing CdTe NCs and then by CE directly on the film before (black line) and after (blue line) the annealing step at $200^{\circ} \mathrm{C}$. The amount of $\mathrm{Hg}$ after the exchange directly on the film, as measured by both ICP-OES and SEM-EDS was $10 \%$, while after the annealing it was $\sim 1 \%$.

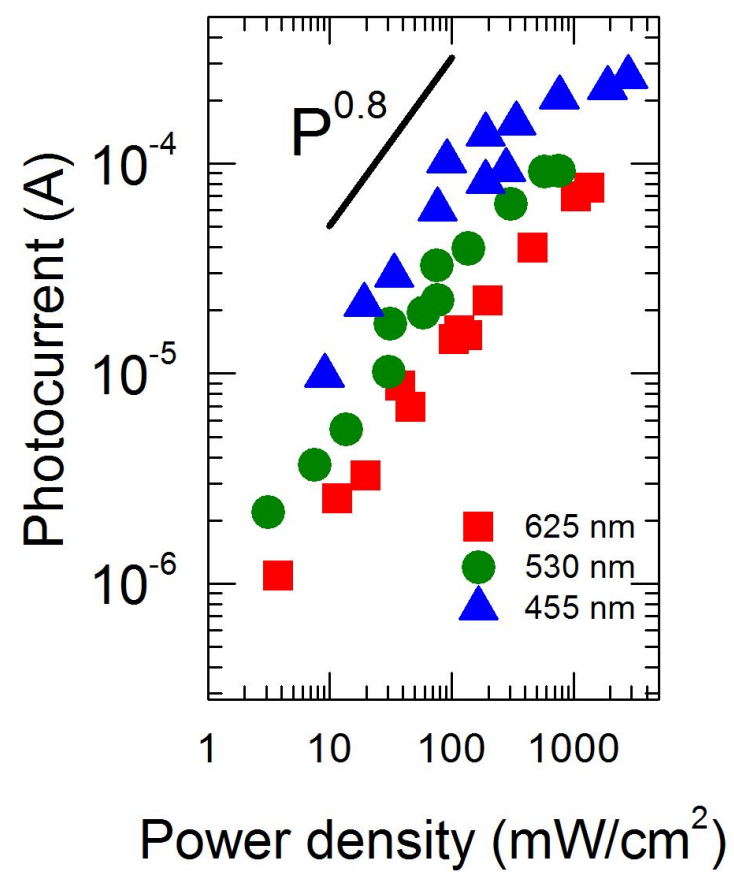

Figure S14. Photocurrent as a function of power density at different photoexcitation wavelengths: $455 \mathrm{~nm}$ (blue), $530 \mathrm{~nm}$ (green) and $625 \mathrm{~nm}$ (red). The continuous, black line marks power law dependence $\mathrm{P}^{\mathrm{o} .8}$. 


\section{Detectivity estimation}

The best theoretical value for the detectivity can be estimated from the lower limit to the noise, calculated including thermal and shot noise. The noise spectral density in these cases are:

$$
\begin{aligned}
S_{\text {th }} & =\sqrt{\frac{k_{B} T}{R_{\text {dark }}}} \\
S_{\text {shot }} & =\sqrt{2 e I_{\text {dark }}}
\end{aligned}
$$

Where $R_{d a r k}$ and $I_{d a r k}$ are dark resistance and current respectively, $T$ is the temperature, $k_{B}$ and $e$ the Boltzmann constant and elementary charge, respectively.

Noise equivalent power, for the different contributions, can be calculated as the ratio of the spectral density to the responsivity:

$$
N E P_{i}=\frac{S_{i}}{\mathcal{R}}
$$

Finally, the detectivity is given by

$$
D^{*}=\frac{\sqrt{A}}{\sum_{i} N E P_{i}}
$$

Where $A$ is the device area.

In our device, we have dark current of $1.1 \mathrm{~mA}$ at $10 \mathrm{~V}$, resulting in $\mathrm{NEP}_{\text {tot }}=3.7 \times 10^{11} \mathrm{~W} \mathrm{~Hz}^{-1 / 2}$ at the maximum responsivity with blue light.

However, an experimental estimation would be more reliable. In this work, we used a numerical estimation of the noise from a current-time signal acquired in the dark, using the same acquisition setup and during the same measurement run of the photocurrent measurements. This procedure avoids the use of additional instruments that may change the noise performance of the whole system. Furthermore, this procedure is also adequate to estimate the noise at the low frequency, of operation of our device. The noise power spectral density is calculated via Fast Fourier Transform (FFT, see e.g., Allen, Mills, "Signal Analysis: Time, Frequency, Scale, and Structure”, 2004, chap. 9). The result is show in the Figure $\mathrm{S}_{15}$, together with the result of the Welch's average periodogram method for comparison (implemented in the psd package of the Python package matplotlib v. 3.1.2, doi: 10.5281/zenodo. 3563226 ).

It can be observed that the noise reaches the flat (white) noise floor at very low frequencies, suggesting that, besides the $1 / \mathrm{f}$ noise in the device, also the setup is contributing to the noise. With the resulting spectral noise density, estimating a value of $\sim 2 \times 10^{-7} \mathrm{~A} \mathrm{~Hz}^{-1 / 2}$ at low frequency in our device we have a NEP of $4 \times 10^{-7} \mathrm{~W} \mathrm{~Hz}^{-1 / 2}$ (blue light) and $10 \times 10^{-7} \mathrm{~W} \mathrm{~Hz}^{-1 / 2}$, corresponding to a detectivity of $\sim 9 \times 10^{4}$ Jones and $2 \times 10^{4}$ Jones for blue and red light, respectively. 


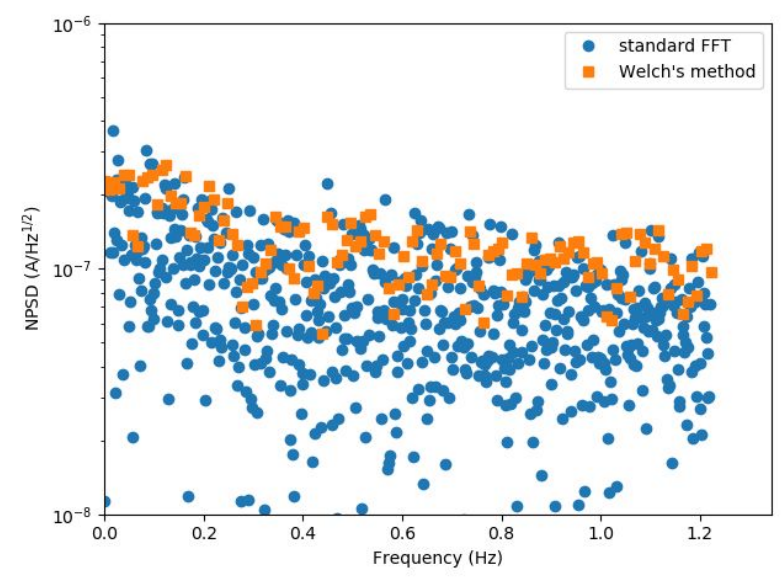

Figure S15. Noise power spectral density (NSPD) for a current-time acquisition, calculated from the FFT or with the Welch's periodogram method. 